\title{
Standardized Evaluation of 2D-3D Registration
}

\author{
Everine B. van de Kraats $^{1}$, Graeme P. Penney ${ }^{1}$, Dejan Tomaževič² \\ Theo van Walsum ${ }^{1}$, and Wiro J. Niessen ${ }^{1}$ \\ 1 Image Sciences Institute, University Medical Center Utrecht, The Netherlands, \\ \{everine, theo, wiro\}@isi.uu.nl, http://www.isi.uu.nl \\ 2 Faculty of Electrical Engineering, University of Ljubljana, Slovenia
}

\begin{abstract}
In the past few years a number of 2D-3D registration algorithms have been introduced. However, these methods have not been directly compared or only work for specific applications. Understanding and evaluating their performance is therefore an open and important issue. To address this challenge we introduce a standard evaluation method, which can be used for all types of methods and different applications. Our method uses the geometry of the 3D Rotational Xray (3DRX) imaging system in combination with $3 \mathrm{D}-3 \mathrm{D}$ registration for attaining a highly accurate ground truth for $2 \mathrm{D}$ multiple X-ray to $3 \mathrm{D}$ $\mathrm{MR} / \mathrm{CT} / 3 \mathrm{DRX}$ registration. The data and ground truth transformations will be made available on the Internet. Furthermore, we propose starting positions and failure criteria to allow future researchers to directly compare their methods. As an illustration, the proposed method has been used to evaluate the performance of two 2D-3D registration techniques, viz. a gradient-based and an intensity-based method, in spinal applications.
\end{abstract}

\section{Introduction and Background}

2D-3D registration has been proposed to help in a number of clinical areas, such as radiotherapy planning and treatment verification, spinal surgery, hip replacement, neurointerventions and aortic stenting.

Several researchers have described and evaluated such methods, but the results are not directly comparable. The use of different datasets, different starting positions, different failure criteria and different error calculation methods hampers direct comparison. To address the challenge of comparing 2D-3D registration algorithms, we introduce a standard validation method, which can be used for different algorithms and different applications. In the field of rigid 3D$3 \mathrm{D}$ registration, the availability of the Vanderbilt dataset has already shown the importance of a common database, validation statistics, and error measure for comparison of multiple algorithms [1].

As an illustration of the method we focus on registering images of vertebral bodies using two registration algorithms: an intensity-based method [2] and a gradient-based method [3]. 


\section{Validation Method}

In the following paragraphs prerequisites for 2D-3D registration methods, the ground truth, accuracy determination, capture range and starting positions, and data preparation of the validation method will be described in detail.

Prerequisites for 2D-3D Registration Methods. The presented evaluation method assumes that the intrinsic parameters of the X-ray device are known and that the $\mathrm{X}$-ray images are corrected for distortion. Therefore six degrees of freedom are left in the 2D-3D registration problem, which are the parameters that describe the position of the 3D volume in space: translations in $x, y$ and $z$ directions $\left(T_{x}\right.$, $\left.T_{y}, T_{z}\right)$ and the orientation of the 3D volume in space: rotations around $x-, y$ and $z$-axis $\left(R_{x}, R_{y}, R_{z}\right)$.

Ground Truth. Data is acquired with a clinical floor-mounted 3D Rotational X-ray (3DRX) C-arm system (Integris BV5000, Philips Medical Systems, Best, The Netherlands). During an 8 second run of 180 degrees around the patient the 3DRX system acquires 100 projection images, which are used to reconstruct a high resolution 3D volume using a filtered-back projection reconstruction technique [4].

The C-arm system needs to be calibrated for 3DRX imaging [5]. Two calibration runs are required: one run to determine the image intensifier distortion and the position of the focal spot of each projection and a second run to establish the projection parameters of the different projections. The accuracy of the derived geometrical parameters for this calibration method was demonstrated in [6], where the three-dimensional image quality was evaluated in terms of spatial resolution and geometrical accuracy. It was shown that the system can obtain an almost isotropic three-dimensional resolution of up to $22 \mathrm{lp} / \mathrm{cm}$ (line pairs per $\mathrm{cm})$. Furthermore, it was shown that the three-dimensional geometrical accuracy is in the order of the spatial resolution of the resulting images.

Since the position of the X-ray images with respect to the reconstructed volume is known from the calibration procedure and since the system is calibrated for distortion [7] of the X-ray images, we can use the 3D volume and the 100 corresponding distortion corrected X-ray images as a ground truth dataset. The use of the standard system calibration enables the acquisition of ground truth datasets for all kinds of anatomical structures without extra or online calibration.

Ground truth registrations for other modalities can be obtained by registering corresponding CT or MR data to the 3DRX data; thereby indirectly obtaining the transformation to the X-ray images. Voxel based 3D-3D registration methods have been described thoroughly in literature and can be performed to subvoxel accuracy. In a previous study we showed that, for spinal images, registration of MR data to 3DRX data based on maximization of mutual information is at least as accurate as marker-based registration [8].

Accuracy of Registration. The accuracy of the registration defines how well the objects of interest coincide after registration. It is measured using the target 
registration error (TRE) 9]. The 'targets' to be used in the TRE calculation can be predefined locations (either fiducials or landmarks), surface points, or arbitrary chosen points inside a region of interest.

We propose to use a set of evenly distributed points in a region centered around the object of interest to determine a mean TRE (mTRE) over a region. For all points $p_{i}$ on a regular grid $P$ inside the region, the distance between the point $p_{i}$ transformed with $T_{\text {ground }}$, the ground truth registration, and the same point transformed with $T_{\text {reg }}$, the transformation determined by the registration algorithm, is computed. The average distance between the points defines the mTRE:

$$
\operatorname{mTRE}\left(P, T_{\text {reg }}, T_{\text {ground }}\right)=\frac{1}{n} \sum_{i=0}^{n}\left\|T_{\text {reg }} p_{i}-T_{\text {ground }} p_{i}\right\| .
$$

Capture Range and Starting Positions. The capture range defines the range of starting positions from which the algorithm finds a sufficiently accurate transformation. Two factors are involved here: the definition of a misregistration, and the fraction of allowed misregistrations.

Several approaches are possible for the determination of the initial distance to the ground truth of a starting position. We choose to use the mTRE at the starting position as a measure for the initial distance. By using the same quantity for initial distance and final result, a direct comparison of the effect of the algorithm is possible.

The starting positions are offsets from the ground truth position centered around a specified 'center of rotation' per object of interest. Given our definition of capture range, and the use of the initial mTRE as the distance measure for the registration starting positions, the starting parameter values must be generated such that several transformations are within several ranges of mTRE. This is achieved by:

1. First choosing intervals for the starting position distance, e.g. 0-1, 1-2 mm.

2. Then, for each interval, for each of the six transformation parameters, the range is determined that will yield a mTRE less than or equal to the interval upper bound. E.g. for a starting mTRE between 1 and $2 \mathrm{~mm}$, the range for each translation is $0-2 \mathrm{~mm}$. Since the region used to determine the starting mTREs is not always cubic, rotations around different axes do not have the same effect on the mTRE. This is taken into account by determining the angle of rotation that results in an mTRE of $1 \mathrm{~mm}$ for each individual rotation. For the next intervals small angle approximation is used which implies that rotations are assumed to linearly relate to the resulting mTRE.

3. Next, for each interval, transformations are generated, where the transformation parameters are chosen randomly (uniformly distributed) from their predetermined range. Subsequently, the mTRE of the composite transformation is determined, and if that mTRE is within the interval it is kept, otherwise the transformation is discarded.

The last step is continued until each interval contains a sufficient number of starting positions. 
Table 1. Parameter values for the registration methods.

\begin{tabular}{lcc}
\hline \multicolumn{3}{c}{ Gradient-based } \\
\hline Parameter & 3DRX & $\mathrm{CT}$ \\
\hline$(\sigma=$ Blur volume $)$ & 0.5 & 0.3 \\
$(\sigma=$ Blur X-ray $)$ & $0.5 / 1.0$ & $0.5 / 1.0$ \\
SampleStep & $1.0 \mathrm{~mm}$ & $1.0 \mathrm{~mm}$ \\
Threshold & 25 & 18 \\
\hline
\end{tabular}

\begin{tabular}{lcc}
\hline \multicolumn{3}{c}{ Intensity-based } \\
\hline Parameter & 3 DRX & CT \\
\hline MinStep & 0.125 & 0.125 \\
Threshold & $13000 / 11000$ & $7000(\approx 400 \mathrm{HU})$ \\
\hline
\end{tabular}

Data Preparation. Most registration algorithms only use a part of the available image data because only the object of interest is relevant and less data results in lower computational costs. Since some algorithms use regions of interest (ROIs) in the $2 \mathrm{D}$ projection images and others VOIs in the $3 \mathrm{D}$ volume, it is difficult to have exactly the same data input available for both types of algorithms. For the presented evaluation method the VOIs are manually determined in the 3DRX volume. When performing intermodality comparison the VOIs are transformed to the corresponding CT or MR dataset using the ground truth image-based 3D$3 \mathrm{D}$ registration. Furthermore, ROIs are determined in the X-ray images, which are not directly related to the VOIs in the $3 \mathrm{D}$ volumes.

\section{Comparison of Two Methods}

The validation method described above has been applied to compare two previously published methods, viz. gradient-based and intensity-based registration. For both algorithms the original implementations were available, though the intensity-based algorithm had to be adapted so it could use multiple X-ray images simultaneously.

The gradient-based 2D-3D registration method [3] registers 3D volumes to $2 \mathrm{D}$ images by searching for the best match between surface normals in the 3D volume and back projected gradients of the 2D X-ray images. The surface normals and positions are extracted from the 3D image in a preprocessing step. In this preprocessing step the volume is blurred with a Gaussian filter, isotropically resampled and the locations with a gradient magnitude larger than some predefined threshold are extracted. The gradient-based registration method needs the following parameters: volume blur, X-ray image blur, sample step and a gradient threshold. See Table 1 for the parameter values used in this evaluation.

The intensity-based 2D-3D registration method [2] compares digitally reconstructed radiographs (DRRs) to X-ray images using a similarity measure based on gradient difference. The algorithm has two parameters: bone threshold and minimal stepsize. The bone threshold is determined such that the gray values above the threshold correspond to bone tissue projected in the X-ray image. The minimal stepsize is the smallest step that the algorithm takes in the optimization procedure. It is a factor by which the parameter values can change in the optimization procedure. The rotations, which are in radians, need an extra 
Table 2. Image data.

\begin{tabular}{lccc}
\hline Modality & Segment & Resolution & Size \\
\hline CT & 1 & $0.31 \times 0.49 \times 0.31 \mathrm{~mm}^{3}$ & $320 \times 260 \times 320$ \\
& 2 & $0.31 \times 0.49 \times 0.31 \mathrm{~mm}^{3}$ & $280 \times 300 \times 300$ \\
3 DRX & 1 & $0.87 \times 0.87 \times 0.87 \mathrm{~mm}^{3}$ & $256^{3}$ \\
& 2 & $0.52 \times 0.52 \times 0.52 \mathrm{~mm}^{3}$ & $256^{3}$ \\
X-ray & 1 & $0.63 \times 0.63 \mathrm{~mm}^{2}$ & $512^{2}$ \\
& 2 & $0.53 \times 0.53 \mathrm{~mm}^{2}$ & $512^{2}$ \\
\hline
\end{tabular}

factor of 0.02 to make the steps comparable to the translation steps, which are in millimeters. The optimization uses a multi resolution approach, where initially coarse resolution $256 \times 256$ pixel X-ray images are used, and then finer $512 \times 512$ pixel images are used. See Table 1 for the parameter values.

Image Data. Two defrosted segments of a vertebral column, both fixed in foam, were imaged with two modalities: 3DRX and CT. The 3DRX images were obtained with a clinical 3DRX system (Integris BV5000, Philips Medical Systems, Best, The Netherlands). The CT images were made with a clinical 16-detectorrow multislice CT scanner (MSCT, Philips Medical Systems, Best, The Netherlands). Spinal segment 1 consists of 3 thoracolumbal vertebral bodies and segment 2 consists of 5 thoracic vertebral bodies. Some soft tissue around the spinal segments was still present. See Table 2 for details on image sizes and resolutions. The CT images were registered to the corresponding 3DRX images, using an algorithm which maximizes mutual information, to establish the ground truth for the CT-to-X-ray registration.

In the 3DRX volumes VOIs were determined (for vertebral bodies in spinal segment 1 approx. $100 \times 50 \times 100$ voxels, and vertebral bodies in spinal segment 2 approx. $120 \times 50 \times 150$ voxels) that were used by the gradient-based method. These VOIs were transformed to the CT data using the ground truth imagebased 3D-3D registration. In the X-ray images ROIs around the vertebral bodies were determined (approx. $170 \times 70$ pixels) that were used by the intensity-based method. See Fig. 1 for examples of the data with corresponding VOIs and ROIs.

Experiments and Evaluation. For each of the eight vertebral bodies, centers of rotation were determined in the world coordinate system (directly linked to the 3DRX data). In this experiment the starting positions were generated as described in Sect. 2, with 10 starting positions per $1 \mathrm{~mm}$ interval up to $20 \mathrm{~mm}$, resulting in 200 starting positions.

From the 100 acquired X-ray images per spinal segment two images were selected, one anterior-posterior and one lateral, which were used for the registration experiment for both CT and 3DRX to X-ray registration. The mTRE was determined in a VOI of $82 \times 39 \times 82 \mathrm{~mm}^{3}$ centered around a vertebral body (as 

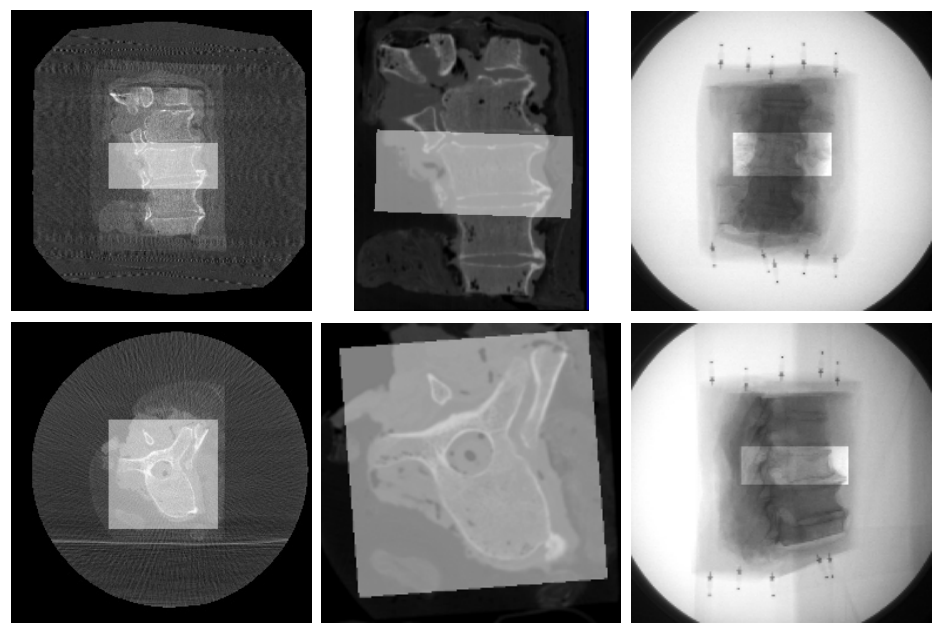

Fig. 1. Left: 3DRX AP and transversal planes with VOIs. Middle: CT AP and transversal planes with corresponding VOIs. Right: two X-ray images with ROIs.

in Sect. 2). Registrations were classified as successful when the end mTRE was smaller than $2 \mathrm{~mm}$. The capture range was defined as the $95 \%$ success range.

\section{Results and Discussion}

We performed 200 registrations per vertebral body (8), per modality (2) and per registration algorithm (2) resulting in a total of 6400 registrations. The average registration time was approx. 25 seconds for the gradient-based method and approx. 9 minutes for the intensity-based method. The algorithms ran on different machines and they were not optimized for speed. The individual registration results, the percentages of successful registrations, the average end mTRE of all the registrations, and the average end mTRE for the successful registrations, all per method and modality, are displayed in Fig. 2.

From the results several conclusions can be drawn. The average error for successful registrations is stable for the gradient-based method, while the intensitybased method has increasing difficulty in finding the optimal position when the initial offset from the ground truth position increases (Fig. 2D,H). This makes failure determination easier for the gradient-based method (Fig. 2A,E) than for the intensity-based method (Fig. 2B,F) because the difference between correct and incorrect registrations is quite large. E.g. when displaying the DRR belonging to the registration position together with the X-ray image, misregistrations can be visually assessed. In both cases the average error is larger for CT to X-ray registration than for $3 \mathrm{DRX}$ to $\mathrm{X}$-ray registration.

The capture ranges for 3DRX and CT respectively are $0-6 \mathrm{~mm}$ and $0-3 \mathrm{~mm}$ for the gradient-based method and 0-4 $\mathrm{mm}$ and $0-3 \mathrm{~mm}$ for the intensity-based 

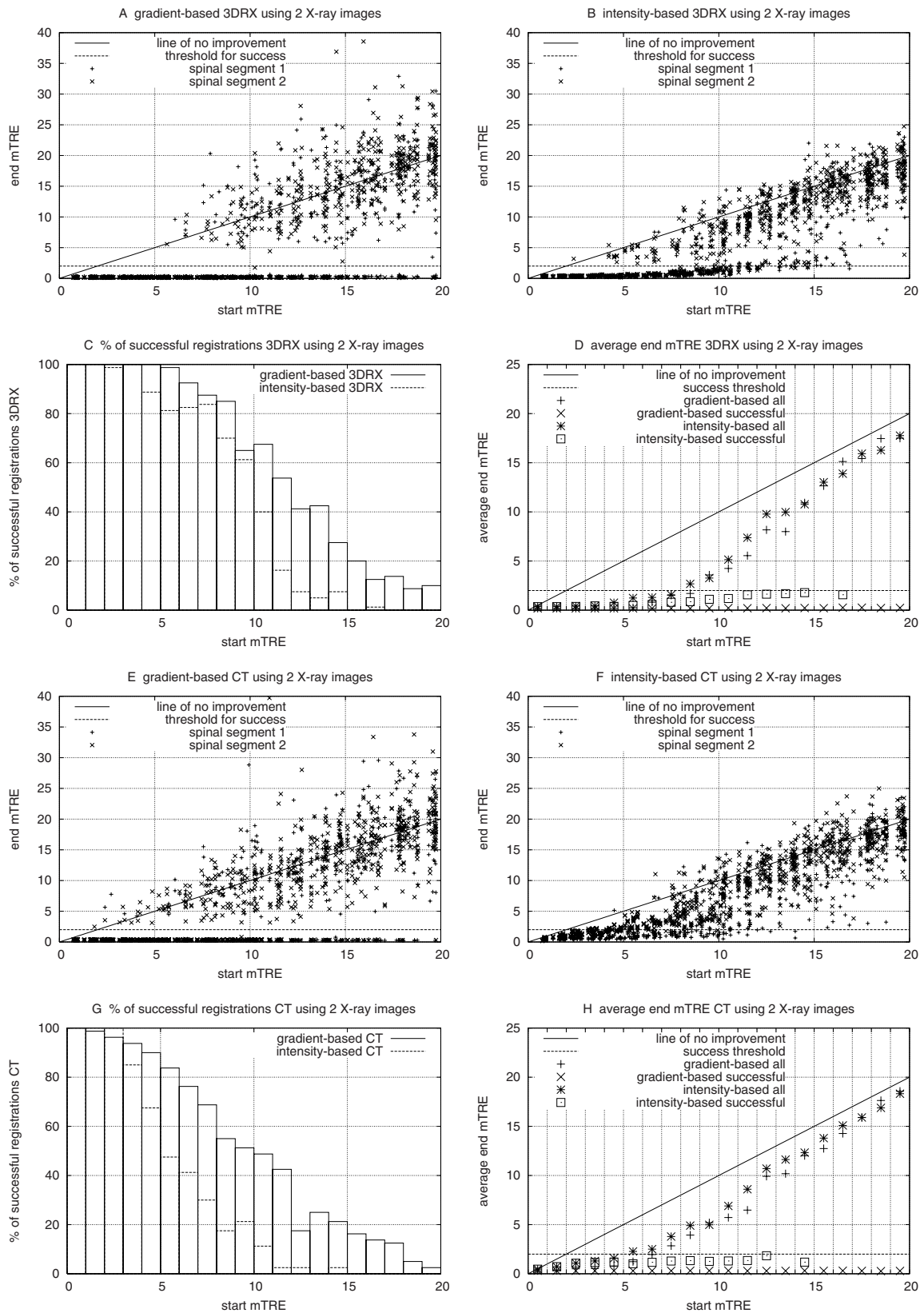

Fig. 2. Results of gradient-based and intensity-based 2D-3D registration, both for 3DRX to X-ray and $\mathrm{CT}$ to X-ray registration. 
method. See Fig. 2C,G. Within the capture range the average end mTREs for 3DRX and CT respectively are $0.19 \mathrm{~mm}$ and $0.38 \mathrm{~mm}$ for the gradient-based method and $0.39 \mathrm{~mm}$ and $0.75 \mathrm{~mm}$ for the intensity-based method.

Better results for 3DRX to X-ray registration could be explained by greater similarity between the 2D and 3D modalities: both X-ray images and 3D volume are produced using X-rays at the same energy (around $60 \mathrm{keV}$ ), whereas CT uses $80 \mathrm{keV}$. Another reason could be an error introduced by the ground truth registration between $\mathrm{CT}$ and 3DRX data.

\section{Conclusion}

The accurate ground truth for 3DRX data and X-ray data in combination with 3D-3D CT/MR-to-3DRX registration, results in a ground truth for objective intermethod and intermodality 2D-3D registration comparison, as is shown in this paper for 2D-3D spine registration.

The ground truth datasets for MR, CT and 3DRX data and corresponding $\mathrm{X}$-ray images, along with starting positions and centers of rotations will be made available on the web. In the future, other applications and anatomical regions can be included in this database.

\section{References}

1. West, J., et al.: Comparison and evaluation of retrospective intermodality brain image registration techniques. J Comput Assist Tomogr 21 (1997) 554-566

2. Penney, G., et al.: A Comparison of Similarity Measures for Use in 2-D-3-D Medical Image Registration. IEEE Trans Med Imaging 17 (1998) 586-595

3. Tomaževič, D., et al.: 3-D/2-D Registration of CT and MR to X-Ray Images. IEEE Trans Med Imaging 22 (2003) 1407-1416

4. Grass, M., et al.: Three-dimensional reconstruction of high contrast objects using C-arm image intensifier projection data. Comput Med Imaging Graph 23 (1999) 311-321

5. Koppe, R., et al.: 3D Vessel Reconstruction Based on Rotational Angiography. In: CAR. (1995) 101-107

6. Rasche, V., et al.: Performance of image intensifier-equipped X-ray systems for three-dimensional imaging. In: CARS. (2003) 187-192

7. Haaker, P., et al.: Real-time distortion correction of digital X-ray II/TV-systems: an application example for Digital Flashing Tomosynthesis (DFTS). Int J Card Imaging 6 (1990) 39-45

8. van de Kraats, E.B., et al.: Noninvasive Magnetic Resonance to Three-Dimensional Rotational X-ray Registration of Vertebral Bodies for Image-Guided Spine Surgery. Spine 29 (2004) 293-297

9. Fitzpatrick, J.M., et al.: The Distribution of Target Registration Error in RigidBody Point-Based Registration. IEEE Trans Med Imaging 20 (2001) 917-927 